\title{
Speed-up of SOLPS-ITER code for tokamak edge modeling
}

E Kaveeva $^{1}$, V Rozhansky ${ }^{1}$, I Senichenkov ${ }^{1}$, I Veselova ${ }^{1}$, S Voskoboynikov $^{1}$, E Sytova $^{1,2}$ X Bonnin ${ }^{2}$, D Coster ${ }^{3}$

${ }^{1}$ St Petersburg State Polytechnical University, Polytechnicheskaya 29, 195251 St Petersburg, Russia

${ }^{2}$ ITER Organization, Route de Vinon-sur-Verdon, CS 90 046, 13067 St. Paul Lez Durance

Cedex, France

${ }^{3}$ Max-Planck Institut fur Plasmaphysik, EURATOM Association, D-85748 Garching,

Germany

E-mail: kaveeva@spbau.ru

Keywords: SOLPS-ITER, tokamak edge modeling

\begin{abstract}
Account of drifts and currents dramatically decreases the accessible time step for the integration of time dependent equations of SOLPS-ITER code for edge modeling. Running the code with sophisticated EIRENE Monte-Carlo model for neutrals and large number of fluid equations for multiple ion species makes the computation time unacceptably long. In the paper the main mechanisms leading to the time step limitations caused by drifts are discussed. Several methods of the suppression of these mechanisms are suggested and the results of numerical scheme tests with the applied corrections are presented. Application of these schemes decreases the time of convergence to steady state solution by more than an order of magnitude.
\end{abstract}

\section{Introduction}

Understanding of edge plasma performance and divertor exhaust is crucial for operation of ITER and other tokamaks. Traditionally this is done by transport codes like SOLPS and others, based on Braginskii model for parallel transport, experimentally based description of anomalous transport and Monte-Carlo model for neutral transport. In the early versions of SOLPS[1,2] selfconsistent electric fields, drifts and currents were ignored. These effects were introduced into the version which is known as SOLPS5.0 [3]. The physics of the edge plasma with drifts is treated much better by the new version [4], however one has to pay a price by slower convergence of the 
code. Later modifications - SOLPS5.2 [5] and its upgrade by the ITER Organization to form a new package, SOLPS-ITER [6,7] still demonstrate low convergence.

Account of drifts and currents dramatically decreases the accessible time step for the integration of time dependent equations of the code. Running the code with sophisticated EIRENE Monte-Carlo model for neutrals and large number of fluid equations for multiple ion species makes the computation time unacceptably long.

In the present paper the mechanisms leading to the time step limitations in SOLPS-ITER are analyzed as well as the ways to relax these limitations. The numerical instability driven by drifts is associated with poloidal redistribution of particles inside the separatrix by $\vec{E} \times \vec{B}$ drift in combination with modification of the radial electric field by diamagnetic currents. It can be overcome by implementation of one of two algorithms. The first method uses artificial slowing down of poloidal density and temperatures redistribution. In the second method equations are modified to get faster convergence to the solution close to the true one, which then is used as an initial approximation for convergence to the true solution. Application of these schemes decreases the time of convergence to steady state solution by more than an order of magnitude. Additional ways to improve convergence by introducing artificial particle sources and artificial increase of the time derivatives are also suggested.

Without the suggested methods for ITER parameters the time step with drifts is of the order of $10^{-8} \mathrm{~s}$, for AUG parameters the time step with drifts is $10^{-7} \mathrm{~s}$. It was found that the limiting value of the time step is associated with the value of the artificial anomalous conductivity $\sigma_{A N}$. The artificial current $j_{y}^{(A N)}=\sigma_{A N} E_{y}$ was first introduced in the current balance equation to increase its order to 2 in the $y$ direction [3]. After the addition of perpendicular viscosity current the order of equation increased to 4 [5], but the anomalous current was retained for convergence purposes. The limiting time step increases approximately linearly with the value of $\sigma_{A N}$. The possible solution to keep large anomalous conductivity is unacceptable, since the realistic solution for the electrostatic potential demands a condition $\sigma_{A N}<\sigma_{\perp}^{N E O}[8]$. Here $\sigma_{\perp}^{N E O}$ is a neoclassical conductivity, which for ITER conditions decreases considerably compared to ASDEX-Upgrade (AUG) [9]. Therefore, the anomalous conductivity in the modeling of ITER should be taken smaller than for AUG. For AUG typical values for accurate description of electric field are $\sigma_{A N}=(1-5) \cdot 10^{-5} e n_{e}$ (here $\sigma_{A N}$, electron charge $e$ and electron density $n_{e}$ are in SI units) while for ITER one should use $\sigma_{A N}=(1-5) \cdot 10^{-7} e n_{e}$. The situation is even more dramatic for ITER runs since the characteristic times are typically longer for larger machines, the simple "diffusive" estimate is $\tau \sim L^{2}$ where $L$ is characteristic length typically proportional to size of the device. Modeling experience shows that typical characteristic times for ITER are 
indeed longer by approximately an order of magnitude. Therefore, the direct calculations for ITER with drifts without any numerical scheme improvements proved unacceptably time consuming, the typical computation times on a 64 processor cluster could reach several years.

Here in section 2 the main mechanism leading to the time step limitations driven by drifts is discussed. The methods of the suppression of this mechanism are suggested and the results of the numerical scheme tests with the applied corrections are presented in sections 3.1-3.2. In sections 3.3-3.4 possible ways of further decrease of computation time based on understanding of typical longest time scales are discussed.

\section{Scheme of numerical instability}

The code SOLPS-ITER is based on the solution of the fluid equations for plasma: the heat balance for electrons is used to obtain electron temperature $T_{e}$; the heat balance for ions is used to find temperature $T_{i}$ common for all ions species; the current balance is used to obtain electrostatic potential $\phi$. The parallel velocity $V_{\| a}$ and the density $n_{a}$ for each ion species $a$ are found using separate equations of the parallel momentum balance and continuity equation correspondingly. The neutral particles are described by either fluid equations or by Monte-Carlo EIRENE package. The latter is much more accurate due to the large (compared to the plasma SOL width) mean free path of the neutrals. Each of fluid equations is discretized using conservative finite volume method on a spatial mesh adjusted to the flux surfaces based on a fixed magnetic equilibrium. A typical mesh for AUG modeling can be found for example in Ref.[3]. Equations are linearized and solved using implicit Euler method. The main source of iteration process instability caused by the fact that although each equation is solved using implicit method, the process of solution in general is not fully implicit. To produce the solution of equation for each quantity $\left(V_{\|}, n, \varphi, T_{e}, T_{i}\right)$ the numerical scheme uses the other quantities calculated previously. Therefore the time step of the iterations is limited by the shortest characteristic time scales of the processes in which interplay of main plasma parameters is important. The convergence to the steady state as well as the description of processes in SOLPSITER time-dependent mode demands calculations for several characteristic time scales of the slowest processes. So the number of necessary iterations can be estimated as a ratio the of longest to the shortest characteristic times.

The more stable algorithm should solve the equations simultaneously and get the quantities at each time step in a self-consistent manner. Indeed, this numerical technique is used in some plasma edge codes, e.g. UEDGE [10]. Unfortunately, this algorithm even for single species plasma increases the solved matrix dimension by a factor 5 , and in the case of multi- 
component plasma the factor is considerably larger. For example for a deuterium plasma with helium and neon impurities, the factor is 29 . The increase in the computational time for a time step together with necessity to reconsider all the procedures of the code, makes this way of code improvement not very attractive.

Additional limitation is associated with coupling of fluid equations of the code to the EIRENE module. Fully implicit scheme including Monte-Carlo modeling of EIRENE is hardly possible, hence UEDGE is limited to using a fluid model for neutrals. Present experience shows that for ITER the limitation of time step associated with EIRENE coupling is about $10^{-6} \mathrm{~s}$. Therefore, the changes in the numerical scheme are desirable increasing the time step limitations for the fluid part of the code up to the EIRENE limitations, without transition to a fully implicit scheme. Such changes can be attained by an artificial increase of the smallest characteristic time scales. Alternative approach, if the steady state solution is looked for, is an artificial reduction of the largest characteristic time scales. The goal of this paper is to suggest a combination of both approaches giving the best results in the framework of the numerical scheme of the code SOLPSITER.

\subsection{Analytical consideration}

The main typical feature of the instability, when the "limiting" time step is exceeded, is oscillation of the electrostatic potential inside the separatrix, from positive to negative values and back, with increasing amplitude from one time step to another. The potential oscillations have no poloidal structure, and they lead to the rise of a big radial electric field changing its sign at each time step. Such behavior resembles geodesic acoustic mode (GAM) formation with a characteristic frequency $\omega \approx \frac{1}{R} \sqrt{\frac{T_{e}+T_{i}}{m_{i}}}$ [11]. The GAM is characterized by the oscillating radial electric field with poloidal and toroidal mode numbers $m=0, n=0$, similar to the electric field of the numerical instability. If the model includes the mechanisms, leading to GAM, the time step of iterations should be smaller than the mode period, $\delta t<<\omega^{-1}$. If this condition is violated, the solution will not be reproduced correctly. Moreover, for an explicit numerical scheme the radial electric field will oscillate with increasing amplitude giving no convergence of the solution.

The simplest description of GAM includes the radial balance of the polarization current and a diamagnetic current, and the particle balance in which the $\vec{E} \times \vec{B}$ drift and parallel transport lead to the density perturbation. The ion particle balance and current balance can be analyzed, leaving all the other quantities unchanged. The temperatures can be assumed flux surface functions. In a simple toroidal co-ordinates $(r, \theta, \varphi)$ of flux surfaces with circular cross section and no Shafranov shift the GAM electric field can be written as $E_{r}=E_{0}+E_{1} \exp (-i \omega t)$ 
and the density distribution as $n=n_{0}+n_{1} \sin \theta \exp (-i \omega t)$. The periodic perturbation of pressure leads to the parallel velocity perturbation: $V_{\|}=V_{0}+V_{1} \cos \theta \exp (-i \omega t)$. The linearization of the continuity equation gives

$-i \omega n_{1}+2 \frac{E_{1}}{R B} n_{0}-\frac{b_{\theta} V_{1}}{r} n_{0}=0$.

Here $\varepsilon=r / R, b_{\theta}=B_{\theta} / B$, and only oscillating contributions corresponding to poloidal $\vec{E} \times \vec{B}$ drift and parallel flow are taken into account. Parallel momentum balance gives

$-i \omega V_{1}+\frac{b_{\theta} c_{s}^{2}}{r} \frac{n_{1}}{n_{0}}=0$

with $c_{s}^{2}=\frac{T_{e}+T_{i}}{m_{i}}$. The pressure perturbation leads to the average diamagnetic current through the flux surface

$$
\left\langle j_{r}^{(d i a)}\right\rangle=-\frac{T_{e}+T_{i}}{B R} n_{1}
$$

which is compensated by the polarization current $j_{r}^{(P)}$,

$$
\begin{aligned}
\left\langle j_{r}^{(d i a)}\right\rangle & =-\left\langle j_{r}^{(P)}\right\rangle \\
\left\langle j_{r}^{(P)}\right\rangle & =-\frac{m_{i} n_{0}}{B^{2}} i \omega E_{1} .
\end{aligned}
$$

Combining Eqs.(1), (2), (4) the GAM frequency can be obtained

$$
\omega^{2}=\frac{c_{s}^{2}}{R^{2}}\left(2+\frac{1}{q^{2}}\right)
$$

where $q=\varepsilon / b_{\theta}$ is safety factor. The limiting time step of iterations in the code describing the GAM physics then would be $\delta t_{\max }^{G A M}=\frac{q R}{c_{s} \sqrt{2 q^{2}+1}}$.

The polarization current is absent in the set of equations solved in SOLPS-ITER. In the description of the evolution of electrostatic potential perturbation it is replaced by the anomalous current:

$$
j_{r}^{\left({ }^{(A N)}\right.}=\sigma_{A N} E_{1} \text {. }
$$

Then, instead of Eq. (4) the current balance is

$$
\left\langle j_{r}^{(d i a)}\right\rangle=-\left\langle j_{r}^{(A N)}\right\rangle \text {. }
$$

Combining Eqs.(1), (2) and (8) the imaginary oscillation frequency can be obtained

$$
\tilde{\omega} \approx-i \frac{2 n\left(T_{e}+T_{i}\right)}{\sigma_{A N}(B R)^{2}},
$$


leading to the exponential decrease for the pressure perturbation and perturbation of $\vec{E} \times \vec{B}$ drift instead of oscillatory solution, $n_{1}, E_{1} \sim \exp (-\gamma t)$ where $\gamma=i \tilde{\omega}$. The characteristic time step for the explicit iteration scheme of SOLPS-ITER is then limited by the decrement of that exponential decrease $\delta t<\gamma^{-1}$, so that

$$
\delta t_{\max } \approx \frac{\sigma_{A N}(B R)^{2}}{n\left(T_{e}+T_{i}\right)} .
$$

The maximal time step is proportional to the anomalous conductivity value. Taking AUG parameters $T_{e} \sim T_{i} \sim 500 \mathrm{eV}, \sigma_{A N}=10^{-5} \mathrm{en}$, one gets $\delta t_{\max }=10^{-7} \mathrm{~s}$ which is close to the values used in our previous simulations. Still, the simple solution to increase $\sigma_{A N}$ cannot be applied in the calculations with drifts directly. The neoclassical conductivity [8]

$$
\sigma_{\perp}^{N E O} \approx \frac{\mu_{i 1}}{B^{2} R^{2}}
$$

should be an order of magnitude bigger than $\sigma_{A N}$, otherwise the result would not reproduce real physics. In the Pfirsch-Schlueter regime $\mu_{i 1} \approx n T_{i} v_{i i}^{-1}$ [12] giving $\delta t_{\max }<<v_{i i}^{-1}$. In the banana regime, typical for ITER plasma, $\delta t_{\max }<<v_{i i} m_{i} q^{2} R^{2} \varepsilon^{-3 / 2} T_{i}^{-1}$ decreases with temperature rise.

In the simulations without drift effects, when $\vec{E} \times \vec{B}$ drift is absent, the parallel velocity and density oscillations leading to numerical instability still exist. In this case the Eq. (1) should be written without the drift contribution

$$
-i \omega n_{1}-\frac{b_{\theta} V_{1}}{r} n_{0}=0
$$

Combining Eq. (2) and Eq. (10) one obtains the characteristic frequency

$$
\omega=\frac{c_{s}}{q R}
$$

and characteristic limiting time step is $\delta t_{\max }^{v}=\frac{q R}{c_{s}}$. The result is not surprising, since $\delta t_{\max }^{v}$ is just the characteristic time for parallel pressure redistribution on the flux surface with the sound speed. Taking into account that the anomalous conductivity should be considerably smaller than the neoclassical conductivity it can be shown that the restriction on the time-step with drifts in any regime (Pfirsch-Schlueter, plateau or banana) is stronger than that without drifts. In the Pfirsch-Schlueter regime $\delta t_{\max }^{v} / \delta t_{\max }>>R v_{i i} / c_{s}$, the value in the r.h.s. is bigger than unity by definition of Pfirsch-Schlueter regime. In the banana regime the rate of the characteristic times in SOLPS-ITER is $\delta t_{\max }^{v} / \delta t_{\max }>>\frac{c_{s} b_{x}}{v_{i i} r} \varepsilon^{3 / 2}$. 
Therefore, the oscillations associated with the drifts start developing at smaller time steps than without drifts. The influence of parallel velocity redistribution on the density equation with drifts can be neglected, it can start limiting the time-step only if drifts are switched off.

\subsection{Numerical realization}

Now let us discuss briefly realization of the instability in the numerical scheme. The equations are solved and corrections are applied in sequence first for density and then for electrostatic potential. Assume no temperature perturbation and initial density deviation from steady-state at the closed flux surface: $n_{(t o p)}>n_{(b o t t o m)}, \delta n / n<<1$, and no perturbation of the radial electric field. In the code SOLPS-ITER the diamagnetic current is changed to $\operatorname{grad} \mathrm{B}$ driven current $\tilde{j}^{(\text {dia })}$ giving the same divergence [3]. For given initial value of density perturbation average current through the flux surface associated with grad B drift is directed inwards. Then solving the equation for potential the quasineutrality demands non-zero average current $\widetilde{j}^{(\text {dia })}$ through the flux surface to be compensated. The anomalous current is the biggest current, which depends directly on the electrostatic potential that can compensate it (the potential equation is solved for $\phi$ with all other variables fixed). So, solving the equation for electrostatic potential one obtains $j^{(A N)}$ directed outwards, and the electric field should get positive.

To solve the continuity equation for density on the next time step and obtain the new density distribution one should take into account the $\vec{E} \times \vec{B}$ drift. For positive electric field $\vec{E} \times \vec{B}$ drift leads to the negative time derivative for $\delta$ n at the top and positive - at the bottom. For sufficiently big time step, we have $n_{(t o p)}<n_{(\text {bottom })}$ at the next time step. If the new values of density have bigger asymmetry between top and bottom of the flux surface, the new $\delta n$ will cause bigger absolute values of $j^{(A N)}$ and electric field on the next time step. The change of the sign of anomalous current leads to the change of electric field direction at each time step. After several time steps the calculations disrupt.

One can estimate the critical time step. The average grad B current at the k-th time step is proportional to the pressure difference between top and bottom of the flux surface, $\delta j^{(d i a)} \sim-\frac{\delta n^{(k)}\left(T_{e}+T_{i}\right)}{B R}$. It should be compensated by anomalous current $\delta j^{(d i a)} \sim-\delta j^{(A N)}$, which gives the radial electric field perturbation, $\delta E^{(k)} \sim \delta n^{(k)} \frac{T_{e}+T_{i}}{B R \sigma_{A N}}$. On the next time step integration of continuity equation for ions gives 
$\delta n^{(k+1)} \approx \delta n^{(k)}-\delta t \cdot n \frac{\delta E^{(k)}}{B R} \approx \delta n^{(k)}-\delta t \cdot n \cdot \frac{T_{e}+T_{i}}{B^{2} R^{2} \sigma_{A N}} \delta n^{(k)}$.

If $\left|\delta n^{(k+1)} / \delta n^{(k)}\right|>1$ - a collapse of the numerical scheme is observed. The estimate for the limiting time step coincides with Eq.(10).

The heat balance is also involved in the oscillations. The pressure perturbation, determining the grad B current through the flux surface, is a combination of temperature and density perturbations. The temperature perturbation oscillates on the time step due to the terms in the heat balance equation including the poloidal $\vec{E} \times \vec{B}$ drift. Due to the solution method implemented in the code, for temperature poloidal perturbation it follows

$\delta T^{(k+1)} \approx \delta T^{(k)}-\frac{5}{3} \delta t T \frac{\delta E^{(k)}}{B R}$

Comparing this equation with Eq.(14) it can be concluded that the temperature perturbations give comparable contributions to the scheme of numerical instability.

\section{Possible solutions for convergence speed-up}

\subsection{Method of intermediate solution}

The idea of the speed-up scheme is to seek first an intermediate solution, which is close to the final true solution running the code with large anomalous radial current. While obtaining this intermediate solution simulations are performed with a large value of anomalous conductivity $\sigma_{A N}$ thus permitting big time step. Simultaneously, neoclassical radial conductivity is increased artificially by introducing artificial large parallel viscosity, to compensate large anomalous current and at the same time to have intermediate profile of radial electric field close to the final one. At this stage other modifications of the equations are introduced in order to avoid unphysical acceleration in the toroidal directon and unphysical change of the radial electron density profiles. At the second stage all modifications are switched off, $\sigma_{A N}$ is reduced to a low level and the true solution is obtained.

The stages of seeking the solution are then the following:

1) Increase anomalous radial conductivity, and modify the equations inside the separatrix. Increase time step to the values limited by processes not associated with electrostatic potential equation convergence. The time step can be of the order of the time step without drifts. Prepare converged state as initial one for step 2.

2) Return to the initial set of equations, decrease anomalous current and time step. Calculate final solution. 
The equations in step 1 are modified as follows. Here the coordinate system $(x, y, z)$ of the code SOLPS-ITER is used with $\mathrm{x}$ - for poloidal, $\mathrm{y}$ - for radial and $\mathrm{z}$ - for toroidal coordinate, $h_{x}=\frac{1}{\|\nabla x\|}$, $h_{y}=\frac{1}{\|\nabla y\|}, h_{z}=\frac{1}{\|\nabla z\|}, \sqrt{g}=h_{x} h_{y} h_{z}$. When anomalous current, which does not exist in reality, is added to the equations, plasma is artificially accelerated toroidally due to $\vec{j}^{(A N)} \times \vec{B}$ toroidal force. To avoid this artificial acceleration it is instructive to introduce simultaneously an additional purely poloidal force $\vec{F}$ acting on ions inside the separatrix, proportional to the radial current so that $j_{y}^{(A N)}=F_{x} B_{z} / B^{2}$. Note that there is no additional radial electron transport, since electron momentum balance is unaffected and neoclassical cancellation of radial drift terms for electrons [13] holds. The ion flow $V_{y}=j_{y}^{(A N)} / e n_{i}$, associated with the artificial force should also be added to the ion particle balance. This flow after integration over the flux surface will be compensated by ion $\vec{E} \times \vec{B}$ and grad B drift flows.

Force $\vec{F}$, having no toroidal component, has the parallel component $F_{\|}=-j_{y}^{(A N)} B B_{x} / B_{z}$ which should be added to the parallel momentum balance. The solution for the neoclassical electric field, obtained from parallel momentum balance [12] will be affected by this force. After averaging of the parallel momentum balance one has

$<\vec{B} \cdot\left(\nabla \cdot \vec{\pi}_{\perp}+n m_{i} \frac{d \vec{V}}{d t}\right)>=-<\vec{B} \cdot \nabla \cdot \vec{\pi}_{\|}>+<\vec{B} \cdot \vec{F}>$

(Here the averaging procedure is $\langle F\rangle \equiv \oint F \sqrt{g} d x / \oint \sqrt{g} d x$, all the notations correspond to $[3,5,8])$

From the neoclassical theory it is known that the average parallel viscosity may be expressed as a function of the radial derivatives of electrostatic potential, ion temperature and density. The general expression, which is valid in all regimes of collisionality, is

$$
\begin{aligned}
& \left\langle\vec{B} \cdot \nabla \cdot \vec{\pi}_{\|}\right\rangle=-v^{(m p)} n m_{i}\left(\frac{B}{h_{y} B_{x}}\left(\frac{\partial \varphi}{\partial y}+\frac{T_{i}}{e n} \frac{\partial n}{\partial y}+k_{T} \frac{\partial T_{i}}{e \partial y}\right)-\left\langle B V_{z}\right\rangle\right) . \\
& v^{(m p)}=\frac{3\left\langle\left(\frac{\vec{B}}{B} \cdot \nabla B\right)^{2}\right\rangle}{\left\langle B^{2}\right\rangle} \frac{\mu_{i 1}}{n m_{i}},
\end{aligned}
$$

where the viscosity coefficient $\mu_{i 1}$ is calculated in [12]. The numerical coefficient $k_{T}$ is $2.7,1.5$ and -0.17 in the Pfirsch-Schlueter, plateau and banana regimes correspondingly. For a tokamak 
with circular cross section and small $\varepsilon=r / R$, where $r, R$ are the radius of flux surface and the major radius accordingly, $v^{(m p)}=\frac{3 b_{x}^{2}}{2 R^{2}} \frac{\mu_{i 1}}{n m_{i}}$.

The 1.h.s. of Eq.(16) can be neglected in the absence of the toroidal spin-up. The neoclassical electric field corresponds to the solution: $\left\langle\vec{B} \cdot \nabla \cdot \vec{\pi}_{\|}\right\rangle=0$, giving a simple equation for the electrostatic potential $\frac{B}{h_{y} B_{x}}\left(\frac{\partial \varphi}{\partial y}+\frac{T_{i}}{e n} \frac{\partial n}{\partial y}+k_{T} \frac{\partial T_{i}}{e \partial y}\right)=\left\langle B V_{z}\right\rangle$. When the artificial force $\vec{F}$ is added to the r.h.s., the equation is modified to:

$$
\left\langle j_{y}^{(A N)} B^{2} B_{x} / B_{z}\right\rangle=-v^{(m p)} n m_{i}\left(\frac{B}{h_{y} B_{x}}\left(\frac{\partial \phi}{\partial y}+\frac{T_{i}}{e n} \frac{\partial n}{\partial y}+k_{T} \frac{\partial T_{i}}{e \partial y}\right)-\left\langle B V_{z}\right\rangle\right) .
$$

In the case of $j_{y}^{(A N)}=0$ we again have the neoclassical solution for electric field $E_{y}=-\frac{\partial \varphi}{h_{y} \partial y}$. To have approximately the same solution, it is necessary to increase artificially the parallel viscosity coefficient $\mu_{i 1}$ so that $\sigma_{A N}<V^{(m p)} n m_{i} /\left\langle B B_{x}^{2} / B_{z}\right\rangle$. This condition is equivalent to $\sigma_{A N}<\sigma_{\perp}^{N E O}$ Eq.(11).

The toroidal force balance determining the radial profile of toroidal rotation is not solved in SOLPS-ITER explicitly. Instead, the toroidal balance can be derived from parallel momentum balance and the expression for radial current, where all drift terms are kept. To get the neoclassical cancellation of the toroidal component of classical viscosity, one should have the same coefficients in the classical viscosity terms in the radial current and in the parallel momentum balance. Therefore the coefficient $\mu_{i 1}$ should be increased also in the classical viscosity contributions to current balance.

The main predictable discrepancy between the true solution and the solution of equations with artificial force contributions and increased viscosity, is a radial scale for transition from SOL to neoclassic electric field. In [14] it is shown that this scale is $\delta \sim\left(\frac{B^{2} r^{2} \eta}{B_{x}^{2} \mu_{i 1}}\right)^{1 / 2}$, where $\eta$ anomalous perpendicular viscosity coefficient. For AUG parameters this scale at the outer midplane is several $\mathrm{mm}$. Artificially increasing classical viscosity one decreases the scale for the electric field radial variation.

When the anomalous conductivity, classical viscosity contributions and time step are simultaneously decreased at the stage 2 , the radial electric field in the separatrix vicinity should change correspondingly. The physical time of the change of electric field can be estimated as time of redistribution of the parallel velocity due to perpendicular viscosity on the scale $\delta$. 
Taking into account that $\eta \approx n m_{i} D$ this time can be estimated as $\tau_{E} \approx \frac{\delta^{2}}{D}$. Substituting $\delta$ the estimate can be made $\tau_{E} \approx \frac{v_{i}}{v_{B}^{2}}$ in the PS regime $\left(v_{B}=\frac{T_{i}^{1 / 2}}{m_{i}^{1 / 2} q R}\right)$ and $\tau_{E} \approx v_{i}^{-1}$ in the banana regime.

This time scale is orders of magnitude smaller than the scales of the diffusive processes, which are required to reach stationary solution at stage 1 . So, the speed-up scheme can give considerable decrease of the computation time. Moreover, the calculations focused on the physical problems associated with processes in the SOL can be stopped at stage 1 . The solution inside the separatrix, which serves as a boundary condition to the problem in SOL, differs at stages 1 and 2 only by details of the poloidal distribution of parallel velocity. For most of the SOL parameters (degree of detachment, heat load distribution at the divertor plates and others) this difference in boundary conditions is insignificant.

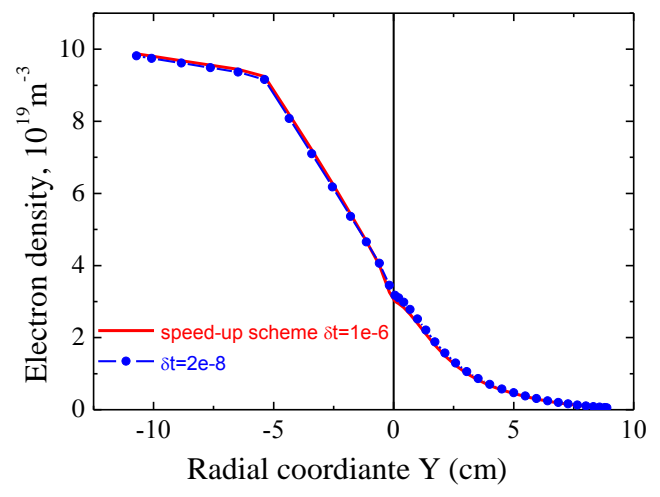

(a)

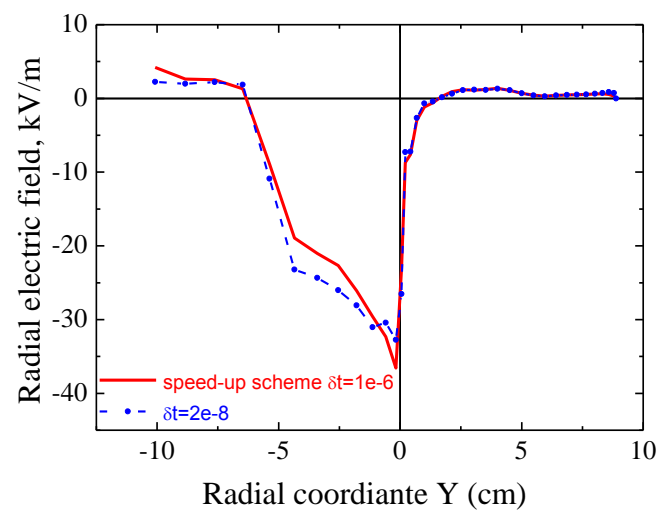

(c)

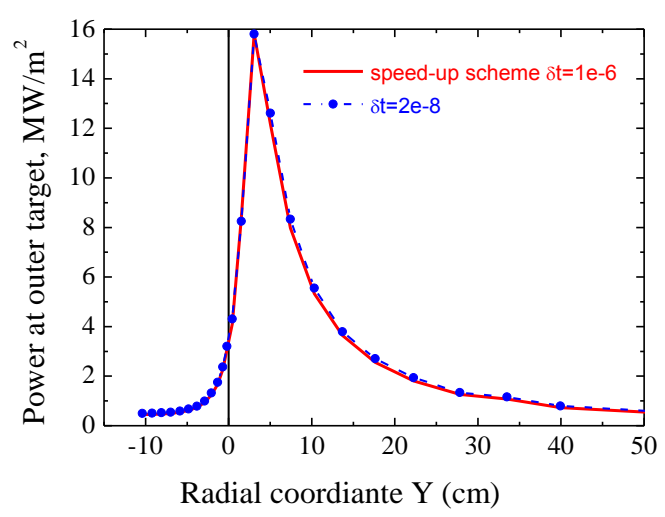

(b)

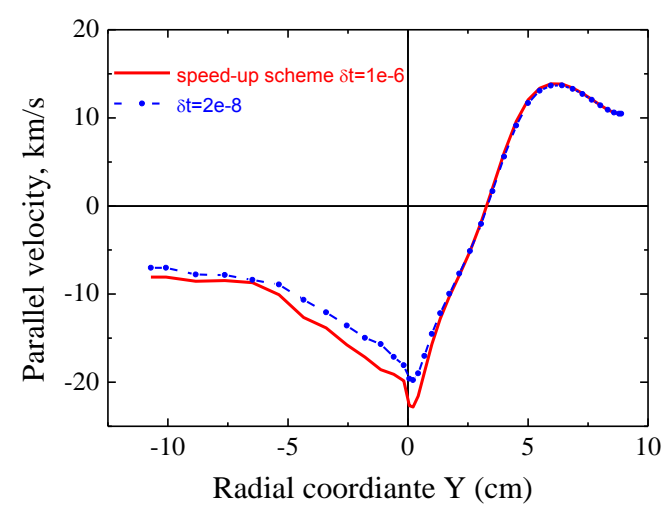

(d)

Fig.1. Solution after initial and final stage of calculations for ITER geometry and typical input parameters. Electron density at the outer midplane (a); energy flow at the outer divertor plate (b); radial electric field at the outer midplane (c); parallel velocity at the outer midplane (d). 
The proposed scheme was tested for ITER simulations. The simulations were performed on a mesh 36x90, for H-mode [9], $100 \mathrm{MW}$ power discharge, for deuterium plasma with helium and neon impurity. All the details necessary to reproduce the calculations presented here and further in the text can be obtained from authors. The full master version of the code SOLPSITER is distributed officially by ITER Organization. The preliminary calculations were continued for about two CPU months with the time step $10^{-6} \mathrm{~s}, \sigma_{A N}=4 \cdot 10^{-6} \mathrm{en}$ and with the viscosity coefficient increased by a factor 20 . Then for a CPU month the calculations were performed from the solution converged at step 1 , with the time step $2 \cdot 10^{-8} \mathrm{~s}$, with $\sigma_{A N}=10^{-7} \mathrm{en}$ and with physically correct viscosity coefficient. The comparison of the preliminary calculations results and final calculations results is shown in Fig. 1. It is seen that the radial profiles of electron density and temperature at the outer midplane did not change. The difference in the radial electric field and toroidal rotation inside the separatrix is small but visible, while the density and heat flow at the outer target are almost unaffected.

The temporal evolution of the calculation is routinely traced using the averaged densities, integral flows or particle contents in different regions of the calculation domain. The example of such evolution of the calculation on the stage 2 is shown in Fig. 2. The deuterium density at the separatrix, Fig.2a, redistributes in a time scale of about $v_{i}^{-1} \sim v_{B}^{-1} \sim 1 m s$, at the same time scale at which the electric field can be expected to redistribute. The relative change in deuterium density is moderate at this time scale, about $1.5 \%$. The following increase of deuterium density at the separatrix has the same long characteristic time as in the initial preliminary calculation. This time can be calculated as a rate of a plasma parameter (temperature, density, number of particles in a region ets...), to its time derivative. Characteristic times longer than $1 s$ are typical for physically meaningful solutions, which can be considered as converged. The neon flow across the separatrix, Fig. $2 \mathrm{~b}$, after the transition period of $1 \mathrm{~ms}$ is the only parameter that stabilizes at considerably different level. The detailed analysis shows that the viscosity change from preliminary to final stage of calculations influences the impurity distribution inside the separatrix more than other parameters. The poloidal distribution of the impurities is very sensitive to the details of the main ions parallel velocity distribution. At the same time the drift radial flows of impurities through the flux surface are sensitive to the poloidal distribution of impurities. Therefore the slight change of parallel velocity and electric field in the vicinity of separatrix leads to the impurity density shift in all the core region. The characteristic times of the process are diffusive ones, of the order of $0.1 \mathrm{~s}$. The analysis of the change in drift flows gives the estimate for the expected change of impurity density in the core of about $10 \%$. To obtain it, a calculation time of about three CPU years can be expected, estimating 
5000 iterations per CPU day with the time step $2 \cdot 10^{-8} \mathrm{~s}$ and the discharge evolution time $0.1 \mathrm{~s}$, which demands $5 \cdot 10^{6}$ iterations. The estimate is given for the computer cluster of St Petersburg State Polytechnical University, where the calculation is performed using 56 processors Intel Xeon E5 $(2.6 \mathrm{GHz}, 64 \mathrm{~Gb})$. The full time of discharge evolution, if we calculate it from considerably different initial state, is determined not only by the diffusive time scales in the transport barrier but also by the processes in the SOL and can reach several seconds. In this case the CPU time necessary for full convergence of ITER calculation with time step $2 \cdot 10^{-8} s$ reaches hundred of years.

As one of the future tasks a method of automatic adjustment of multiplication factor for the viscosity, the anomalous conductivity and the time step during the calculation can be discussed, in order to obtain the converged correct solution in a continuous code run.

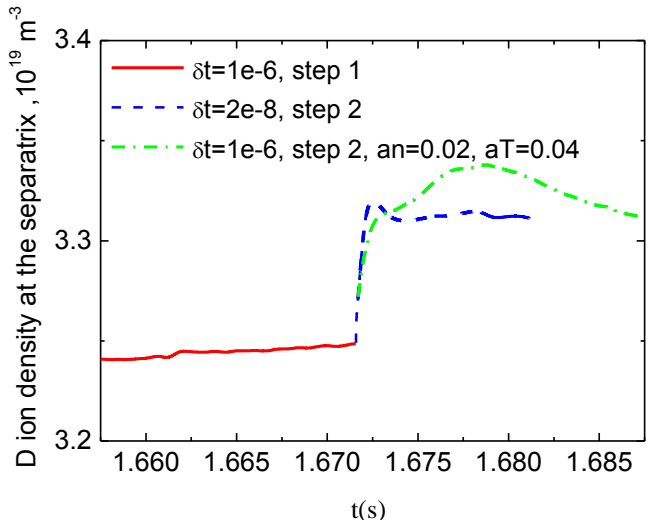

(a)

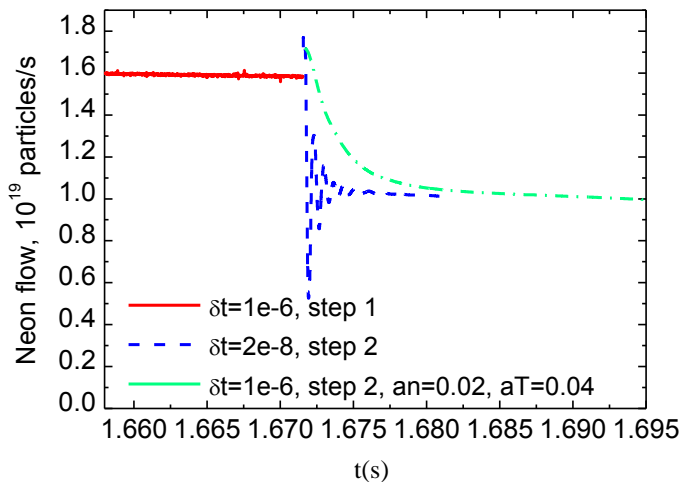

(b)

Fig. 2. Time dependencies of parameters during switch from time step $10^{-6} \mathrm{~s}$, viscosity multiplied by factor 20 , to the time step $2 \cdot 10^{-8} s$, correct viscosity. Switching happens at 1.6715 s. (a) Deuterium ion density at the separatrix, (b) neon flow through the separatrix.

\subsection{Method of partial flux surface averaging}

To relax the time step limitations it is possible to separate the time derivative of average density and heat energy on the flux surface and that of the density and temperature perturbation for the region inside the separatrix. For the average density and stored energy at the flux surface the derivatives are determined by anomalous diffusion and heat conductivity. The diffusive processes are slow $\tau_{D} \sim L^{2} / D$. Taking the transport barrier width and the diffusion coefficient for estimate, we get estimate $\tau_{D} \sim 100 \mathrm{~ms}$ for ITER $\left(L \sim 5 \mathrm{~cm}, D \sim 0.03 \mathrm{~m}^{2} / \mathrm{s}\right)$ and $1 \mathrm{~ms}$ for AUG $\left(L \sim 1.5 \mathrm{~cm}, D \sim 0.2 \mathrm{~m}^{2} / \mathrm{s}\right)$. Therefore one should numerically follow the evolution of average density and temperatures for several slow characteristic time scales to get the converged solution. The instability as shown above is driven by the density and temperature perturbations at 
faster time scales. So it would be reasonable to decrease numerically the time derivative of the density perturbation. The implementation of this scheme in the code is described below.

First, the corrections for plasma component densities $\delta n_{a l, m}^{(k)}(a-$ ion species, $k$ - time step index, $l$ - radial and $m$ - poloidal indexes of numerical grid cells), and for electron and ion temperatures $\delta T_{e l, m}^{(k)}, \delta T_{i l, m}^{(k)}$ are calculated routinely at the time step. Next, inside the separatrix the average corrections:

$$
\begin{aligned}
\left\langle\delta n_{a l}^{(k)}\right\rangle & =\frac{\sum_{m} \delta n_{a l, m}^{(k)} \sqrt{g_{l, m}}}{\sum_{m} \sqrt{g_{l, m}}} \\
\left\langle\delta T_{e l}^{(k)}\right\rangle & =\frac{\sum_{m} \delta T_{e l, m}^{(k)} n_{e l, m} \sqrt{g_{l, m}}}{\sum_{m} n_{e l, m} \sqrt{g_{l, m}}} \\
\left\langle\delta T_{i l}^{(k)}\right\rangle & =\frac{\sum_{a, m} \delta T_{i l, m}^{(k)} n_{a l, m} \sqrt{g_{l, m}}}{\sum_{a, m} n_{a l, m} \sqrt{g_{l, m}}}
\end{aligned}
$$

are calculated on each flux surface and are applied to each cell. Such averaging gives the same increase/decrease of the full number of particles and stored energy in electron and ion components of plasma in the volume between chosen flux surfaces as initial correction. Then, the corrections for densities and temperatures perturbations are calculated and applied with scaling coefficients $\alpha_{a}<1, \alpha_{T}<1, \alpha_{T} \sim \alpha_{a}$. At the beginning of the next time-step the densities and temperatures are:

$$
\begin{aligned}
& n_{a l, m}^{(k+1)}=n_{a l, m}^{(k)}+\left\langle\delta n_{a l}^{(k)}\right\rangle+\alpha_{a}\left(\delta n_{a l, m}^{(k)}-\left\langle\delta n_{a l}^{(k)}\right\rangle\right) \\
& T_{e l, m}^{(k+1)}=T_{e l, m}^{(k)}+\left\langle\delta T_{e l}^{(k)}\right\rangle+\alpha_{T}\left(\delta T_{e l, m}^{(k)}-\left\langle\delta T_{e l}^{(k)}\right\rangle\right) \\
& T_{i l, m}^{(k+1)}=T_{i l, m}^{(k)}+\left\langle\delta T_{i l}^{(k)}\right\rangle+\alpha_{T}\left(\delta T_{i l, m}^{(k)}-\left\langle\delta T_{i l}^{(k)}\right\rangle\right)
\end{aligned}
$$

The modification of correction should not be applied to the partially ionized impurity states that have very strong poloidal density variation inside the separatrix due to big ionization sources and sinks. For such ionisation states the modification of correction can in some cases lead to negative densities. Therefore after the application of modified correction the density is checked to ensure that it does not fall below the limiting value $n_{a \min }$ which is determined by user. In case if it is not determined explicitely, $n_{a \min }=10^{4} \mathrm{~m}^{-3}$. If some of the density values for species $a$ at the flux surface are below the limit after application of modified correction, the modification of 
correction cancels out and the correction is applied without modification for species $a$ at this flux surface.

The correction modification is applied only inside the separatrix in the volume cells (not applied in the narrow boundary cells at the core boundary). The boundary cells should be excluded from the correction due to following reasons. The usual boundary condition is (in its numerical realization) of Dirichlet type. For example for density it is realized through the solution of equation:

$n_{a l, m}^{(k+1)}=n_{a l, m}^{(k)}-\beta\left(n_{a l, m}^{(k+1)}-n_{0 m}\right)$

Where $n_{0}$ is prescribed value and $\beta$ is a big coefficient. If no correction is applied then $n_{a l, m}^{(k+1)}=\frac{n_{a l, m}^{(k)}+\beta n_{0 m}}{1+\beta} \underset{\beta \rightarrow \infty}{\longrightarrow} n_{0, m}$, and that is the required result.

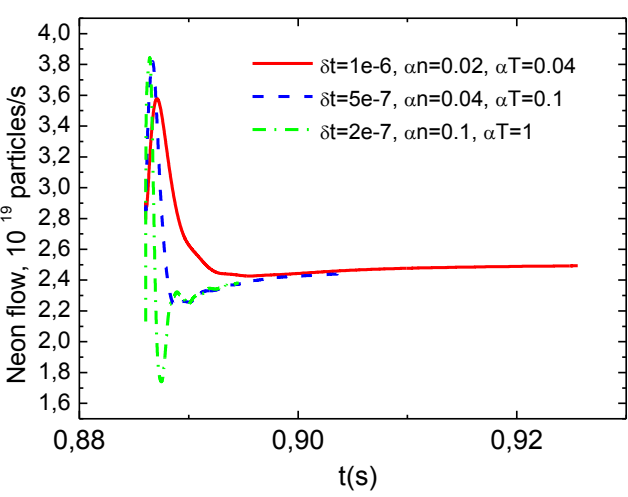

(a)

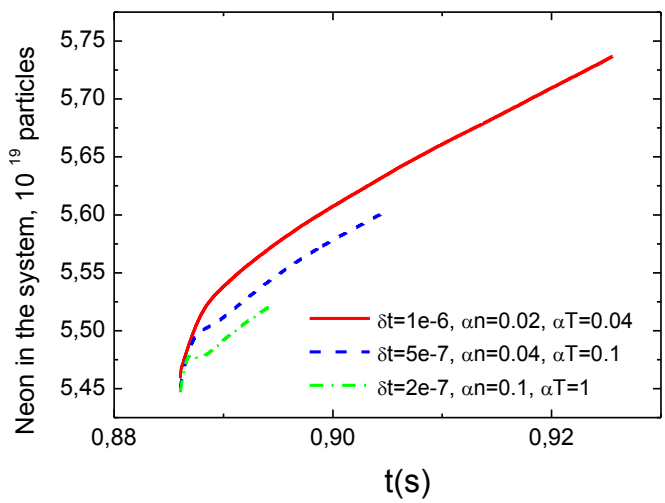

(b)

Fig.3. Time evolution of the neon flow through the separatrix (a), and full number of neon ions in system (b) for ITER modeling with different speed-up parameters.

The correction in volume cells inside the separatrix damps the effect of the redistribution of particles at the flux surface due to the $\vec{E} \times \vec{B}$ drift and permits the increase of time step roughly by a factor $\alpha_{T}^{-1} \sim \alpha_{\text {a main }}^{-1}$ (here "main" is the label for the ion species contributing most to the plasma pressure). In the case $\alpha_{a}=\alpha_{T}=1$ the modification of correction disappears. This scheme does not affect the converged solution. The coefficients $\alpha_{a}$ for small fractions of impurity (the criteria is $\left.n_{a}\left(Z_{a} T_{e}+T_{i}\right)<<n_{a \text { main }}\left(T_{e}+T_{i}\right)\right)$ can be chosen equal to unity, since the time scales for pressure redistribution at the flux surface for small fractions of impurity are long and at the same time the poloidal pressure perturbation influences their neoclassical radial transport. The instability depends on full pressure and will not be affected by these impurities. 
The scheme should not be used for the modeling of the discharges in a state of deep detachment, where the density and pressure perturbation at the flux surfaces next to X-point can be of the order of unity.

The described scheme was tested for the same ITER shot as in the section 3.1 where the time step limitation without corrections was $5 \cdot 10^{-8} \mathrm{~s}$. The time evolution of the discharge at the stage 2, described in section 3.1 was calculated with time step $5 \cdot 10^{-7} \mathrm{~s}$ for $\alpha_{T}=0.04, \alpha_{a}=0.02$. Fig. 2 shows that the time evolution at the small time scales of the order of several milliseconds depends on the speed-up parameters $\alpha_{T}, \alpha_{a}$. Still, finally speed-up scheme tends to the same steady-state result as the calculation with the small time step.

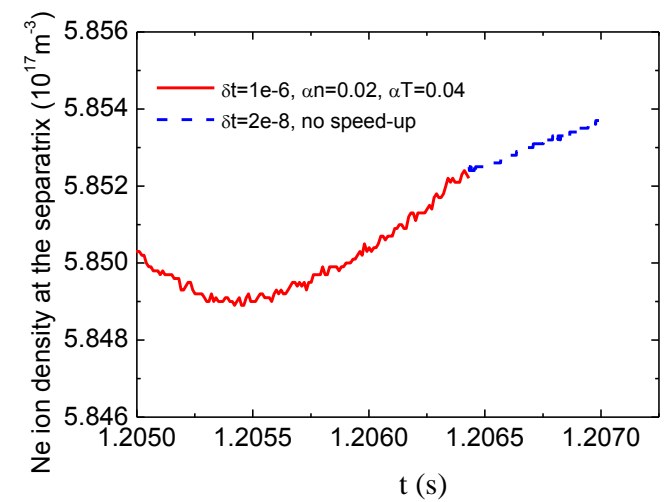

(a)

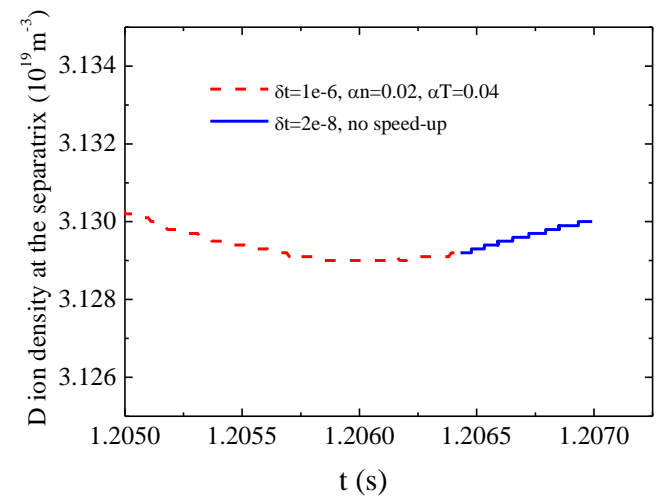

(b)

Fig.4. Time evolution average separatrix density of (a) Neon (b) Deuterium with the speed-up scheme and after the speed-up scheme turning off.

The time evolution at the longer time scales was investigated with three sets of parameters for speed-up scheme, since the time of calculations for the scheme without speed-up was too big. Here, the number of time steps, $4 \cdot 10^{4}$, was fixed, giving a physical time of $40 \mathrm{~ms}$ for the biggest time step $10^{-6} \mathrm{~s}$. The neon puffing was increased by $20 \%$ in the beginning, to investigate the behavior of different speed-up schemes in the case of change in the modeling parameters. The time evolution obtained in a week of calculations is presented in Fig. 3. The time traces in the first $5 \mathrm{~ms}$ are considerably different. Still the flow of neon through the separatrix tends to the same values for all three speed-up parameter sets during the following evolution. The full number of neon ions in the calculation domain, Fig. 4b, differs slightly for different speed-up parameters. This can be explained by the effect of different misbalance between puffing and pumping at the initial stage, for the first $5 \mathrm{~ms}$. Nevertheless, the time derivatives for all three schemes after the first $5 \mathrm{~ms}$ tend to the same value showing the same difference between puffing and pumping. The following evolution of the discharge is the same for all three schemes but "delayed" by about $7 \mathrm{~ms}$ for calculation with time step $2 \cdot 10^{-7} \mathrm{~s}$ 
comparatively to calculation with time step $10^{-6} \mathrm{~s}$. Taking into account typical characteristic time about $100 \mathrm{~ms}$ for ITER core and several seconds for ITER SOL, this delay in time evolution is insignificant.

The test of converged solution was performed at the next step. The speed-up scheme was applied with coefficients $\alpha_{T}=0.04, \alpha_{a}=0.02$ for time step $10^{-6} \mathrm{~s}$ and then after the convergence of calculation the time step was decreased to $2 \cdot 10^{-8} s$ and speed-up scheme turned off. The calculation gives characteristic times of about 2 seconds for the evolution of the solution after the turn-off of the speed-up in Fig. 4, which corresponds to the converged state. The evolution of calculation shows no great change during the speed-up turning off.

The described scheme proved to be robust for ITER conditions. The time step limitation from EIRENE coupling is $10^{-6} \mathrm{~s}$, therefore further increase of convergence speed is limited by EIRENE. Since on a large time scale the evolution of the system is the same with or without speed-up the increase of the time step leads to the proportional decrease of the necessary number of time steps and therefore to the decrease of CPU time (each time step needs almost the same CPU time), in described case of ITER by a factor 50 .

Another set of tests was made for AUG conditions. The possible increase in the time step was from $2 \cdot 10^{-7} \mathrm{~s}$ for no speed-up coefficients up to $10^{-5} \mathrm{~s}$. The AUG shot evolution was traced after the drifts turning on and after the change in pumping rate. In the first case tracings for speed-up coefficients $\alpha_{T}=\alpha_{a}=0.04$ and time step $2.5 \cdot 10^{-6} \mathrm{~s}$ were compared to tracings for $\alpha_{T}=\alpha_{a}=0.01$ and time step $10^{-5} \mathrm{~s}$, Fig.5.

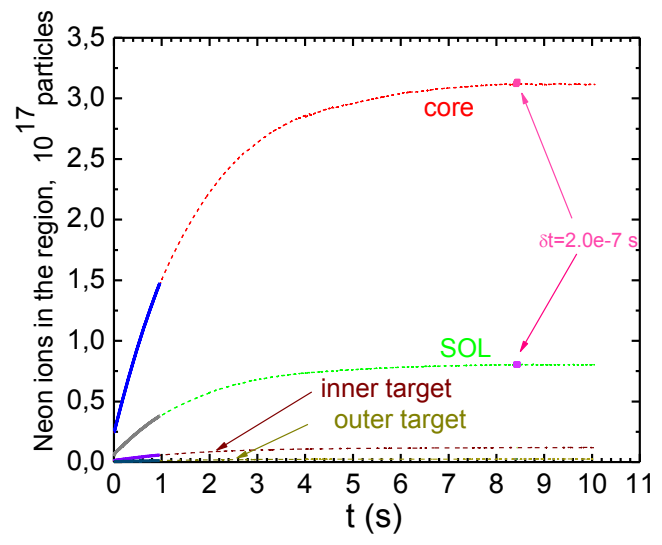

(a)

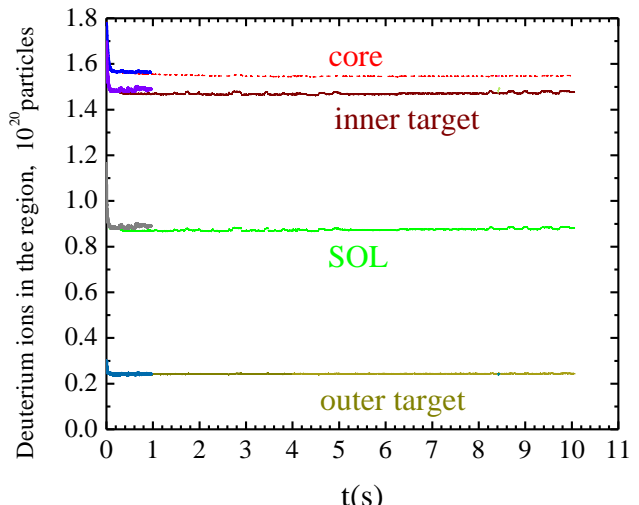

(b)

Fig. 5. The full number of particles in different regions during the evolution of the AUG discharge with neon impurity after the drifts turning on. Speed-up coefficients $\alpha_{T}=\alpha_{a}=0.04$ and time step $2.5 \cdot 10^{-6} \mathrm{~s}$ - solid lines; speed-up coefficients $\alpha_{T}=\alpha_{a}=0.01$ and time step $10^{-5} \mathrm{~s}-$ dashed lines. (a) Neon (b) Deuterium. 


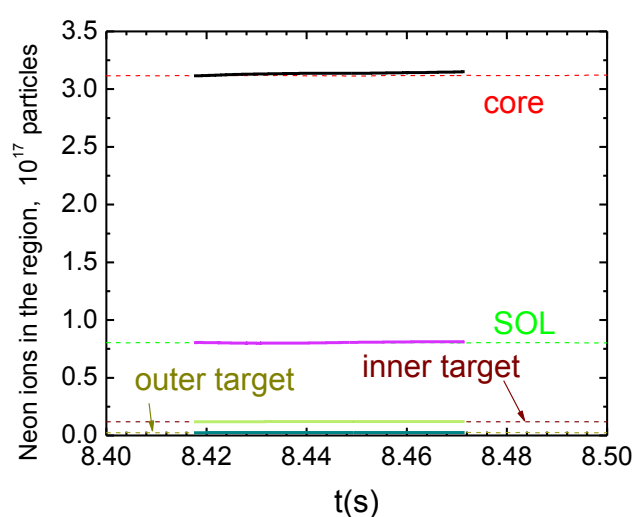

(a)

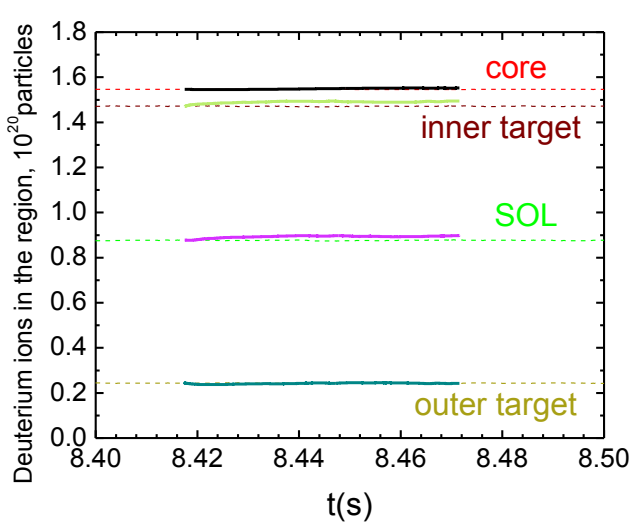

(b)

Fig. 6. The full number of particles in different regions during the evolution of the discharge with the speed-up scheme (dashed lines) and after the speed-up scheme turning off (solid lines). (a) Neon (b) Deuterium

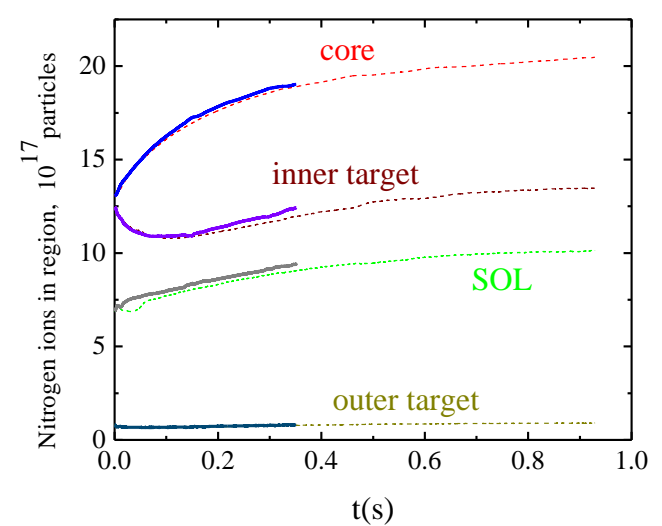

(a)

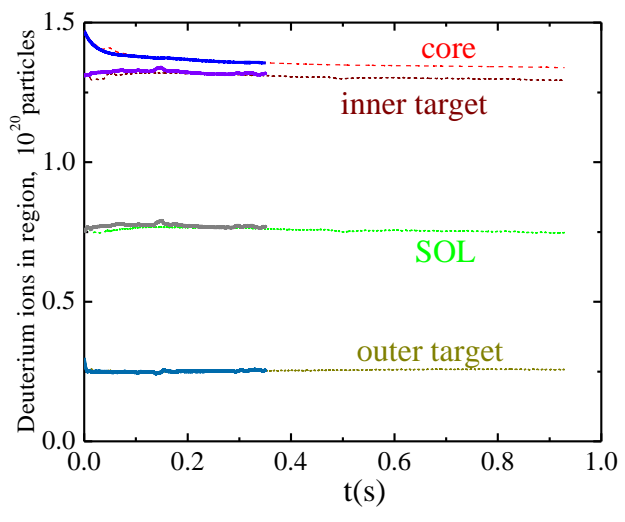

(b)

Fig. 7. The full number of particles in different regions during the evolution of the discharge after increase of pumping. Speed-up coefficients $\alpha_{T}=0.5, \alpha_{n}=0.1$ and time step $10^{-6} s-$ solid lines); $\alpha_{T}=0.05, \alpha_{n}=0.02$ and time step $10^{-5} s$-dashed lines. (a) Nitrogen (b) Deuterium

The two sets of speed-up parameters give the same evolution of the calculation with the only difference that the steady state solution for time step $10^{-5} \mathrm{~s}$ can be obtained 4 times faster in terms of calculation time. The full convergence without the speed-up scheme would take correspondingly about $10^{2}$ times longer, for our calculation capacities it would be several years. Therefore, to make the test of converged solution it was obtained in a month of calculation with speed-up parameters $\alpha_{T}=\alpha_{a}=0.01$ and the time step $10^{-5} \mathrm{~s}$ and then the calculation was continued without the speed-up scheme and with small time step $2 \cdot 10^{-7} \mathrm{~s}$. The points in time where the calculations were continued are shown in Fig. 5(a) with arrows on tracings. The time tracings of the calculation before and after the change of the time step are shown in Fig.6. The 
calculation without the speed-up scheme shows that the stationary solution indeed does not change if the scheme is turned off.

The evolution of AUG shot with nitrogen impurity is shown in Fig.7 after the change in pumping rate from albedo at the cryopumping surface 0.81 to 0.74 . The tracings for speed-up coefficients $\alpha_{T}=0.5, \alpha_{a}=0.1$ and time step $10^{-6} s$ were compared to tracings $\alpha_{T}=0.05, \alpha_{a}=0.02$ and time step $10^{-5} \mathrm{~s}$. Again for different time steps and different speed-up coefficients the evolution of the full amount of nitrogen and deuterium in the system is almost the same. It can be explained by the smallness of the time of radial electric field redistribution. Even for artificially increased electric field redistribution time, this time is much smaller than the time of the radial density and temperature profile change. So the electric field follows the evolution of the radial density and temperature profile (approximately in a neoclassic manner inside the separatrix [3]) with almost no delay with different coefficients in speed-up scheme. Therefore, we expect this speed-up scheme to be valuable for the time dependent mode of SOLPS-ITER.

All these tests prove the robust behavior of the speed-up scheme applied in SOLPS-ITER with EIRENE treatment of neutrals, which can be recommended for routine application. Corresponding tests were performed in the calculations with fluid neutrals description. In case of pure deuterium both in ITER and in AUG geometry the tests show that the new scheme allows to achieve a tenfold increase of time step in order to get steady state solution of plasma equations with drifts. The tests in AUG geometry were performed also for multispecies plasma. It was found for multispecies plasma with the fluid description of neutrals that impurity behavior depends on the time step even in the case of no speed-up coefficients. The time of convergence to stationary state for impurity increased with the time step, both with and without the application of speed-up coefficients. Still, the converged solutions were the same. It could be concluded that for multispecies plasma with the fluid description of neutrals the effect of application of the speed-up scheme is still positive but it is smaller than for calculations with EIRENE.

\subsection{Method of effective source}

The time step increase above the level $10^{-6} s$ for ITER and $10^{-5} s$ for AUG is problematic due to coupling to EIRENE. This limitation requires about $10^{5}$ time steps for AUG and more than $10^{6}$ time steps for ITER to obtain converged solutions, which for typical cluster performance means between a month and a year of simulations. 
Usually the longest characteristic time determining the convergence to steady-state is a time necessary to balance gas puff and pumping rates. The pumping rate is determined by the number of particles in front of the pumping surface and albedo coefficient at this surface while the puffing is determined by the user as a given inflow. To balance the puffing and pumping in the case, for example, of puffing rise, the system should accumulate enough particles to increase the neutral pressure under the dome or in other location where the pumping surface is situated. In the case of puffing decrease the extra particles should be pumped. The estimate for the characteristic time of this process can be made using a very simple model of the gas reservoir of volume $\mathrm{V}$, with constant pressure and pumping rate proportional to the density. Let in the initial steady state the gas puff to be $\Gamma_{0}$ and it is changed to $\Gamma$ at $\mathrm{t}=0$. The equation for density evolution is:

$V \frac{d n}{d t}=\Gamma-n R$

where $\mathrm{R}$ is a pumping rate coefficient. The solution is

$n=\frac{\Gamma}{R}+\frac{\Gamma_{0}-\Gamma}{R} \exp (-t R / V)$

The characteristic time for density variation is $V / R$, the same time as for pumping of all particles from the volume. To estimate the characteristic time in plasma we can take the rate of full particle amount in the system to the pumping flow, which in ITER is about $1 \mathrm{~s}$. For convergence several such times should pass. For deuterium this estimate is typically several times smaller than for neon due to the neon concentration in the core and upper part of SOL. This estimate should be used with extreme care since the tokamak is a complex non-linear system. For example, degree of detachment can change the pressure under the dome and therefore the pumping efficiency without considerable change in plasma content inside the separatrix. Still for the general understanding of the problem the simplified model is useful.

The characteristic time of pumping-puffing equilibrium is determined by pumping efficiency and in the case of small pumping can be unacceptably long. The process can be accelerated by simultaneous increase of pumping coefficient and puffing flow, since the saturated density depends on their ratio while the characteristic time depends only on $\mathrm{R}$. The natural limit to this approach is smallness of the puffing and pumping flows compared to the sound speed, to be consistent with constant pressure assumption. In nonlinear tokamak case the restrictions are even stronger, to provide generally the same solution the increased puffing should not lead to significant local perturbations of plasma. However, even minimal changes in the solution due to nonlinearity make the solution of problem with increased puffing and 
pumping acceptable only as a preliminary step. On the finalizing step both puffing and pumping should be decreased and calculations continued. Therefore this way is not very promising.

Another possible solution is addition of volumetric artificial source $S$ proportional to the difference between puffing and pumping flows, leading to the equation:

$V \frac{d n}{d t}=\Gamma-n R+S$

where $S=\beta(\Gamma-n R)$. The characteristic time in this case decreases by a factor $(\beta+1)$, while saturated density does not change. For converged solution the artificial source disappears so that we come to the correct solution in one cycle of calculations.

This approach was implemented for SOLPS-ITER. The additional source of main ions and impurities was applied through the multiplication factor on the source $S_{E i r}$, which is passed from EIRENE to fluid part of the code and includes both ionization from neutrals and recombination. Since the source is mainly determined by ionization of the recycled neutrals the full ionization in the volume $\int S_{E i r} d V$ is typically about two or three orders of magnitude bigger than the puffing and pumping flows, so the artificial source implemented as slight increase or decrease of ionization source should not affect the local solution dramatically. The ionization source is localized mainly in the divertor regions, therefore the additional source is distributed mainly there. Both the puffing $\Gamma_{\text {wall }}$ from the wall and the particle flow through the inner core boundary $\Gamma_{\text {core }}$ should be taken into account. The multiplication factor $\tau$ should be calculated as follows:

$$
\tau=\beta \frac{\Gamma_{\text {wall }}+\Gamma_{\text {core }}-\Gamma_{\text {pump }}}{\int S_{\text {Eir }} d V}
$$

where $\beta$ is a numerical coefficient. Flows $\Gamma_{\text {pump }}, \Gamma_{\text {wall }}$ and $\Gamma_{\text {core }}$ are summed over all the charge species of the same nuclei, the former is taken positive if directed from the plasma, while two others are positive when directed into the plasma. The coefficient $\tau$ is then averaged on chosen time scale $t_{0}=50 \delta t$ ( $\delta t$ is a time step of iteration) in order to avoid oscillations of this value due to EIRENE statistical noise in the source and pumping flow:

$$
\tau=\frac{\int_{-\infty}^{t} \tau\left(t^{\prime}\right) \exp \left(\frac{t^{\prime}-t}{t_{0}}\right) d t^{\prime}}{\int_{-\infty}^{t} \exp \left(\frac{t^{\prime}-t}{t_{0}}\right) d t^{\prime}},
$$

restricted by 0.05 in absolute value and finally applied to the EIRENE source. The modified source is $\tilde{S}_{E i r}=S_{E i r}(1+\bar{\tau})$ if $|\bar{\tau}| \leq 0.05$ or $\tilde{S}_{E i r}=S_{E i r}(1+0.05 \bar{\tau} /|\bar{\tau}|)$ if $|\bar{\tau}|>0.05$. 
This source modification was applied to the ITER runs. In several cases it gives the opportunity to decrease calculation time by a factor of 10 . The best results were obtained using this approach with $\beta \approx 10-50$. The proposed scheme was tested for the plasma without material sputtering/re-deposition at the wall. In the case of sputtering and re-deposition, for example for modeling of ITER plasma with beryllium, the scheme should be reconsidered. When the recycling in the divertor region is modified by this artificial source, the puffing to pumping balance can be achieved quickly with accuracy $80-90 \%$ by proper choice of parameter $\beta$. Our experience shows that characteristic time of puffing to pumping balance in typical ITER conditions can be made smaller than typical times of particle redistribution due to diffusion and impurity convection in the SOL.

\subsection{Use of multiplicators}

The perpendicular anomalous diffusion time in the transport barrier and SOL $\tau_{y} \sim L_{y}^{2} / D$ can be estimated for each specific condition of plasma. The impurity redistribution inside the separatrix is determined by neoclassical diffusivity of impurities [12,13], which is typically of the order of or larger than the anomalous diffusion coefficient in the transport barrier. The time of parallel redistribution of the main ions in the SOL is comparatively small - it is determined by the ion temperature redistribution by parallel heat conductivity of ions and characteristic time of pressure redistribution. The characteristic time of parallel transport of impurities can be longer. According to modeling experience the impurities are redistributed in the parallel direction in the SOL by convective flows that are determined by temperature and parallel velocity of the main ions [15]. The redistribution time in this case can be estimated as $\tau_{x} \sim r B /\left(B_{x} V_{\|}\right)$, and for the regions in the far SOL, where parallel main ions velocity $V_{\|}$is small, it can be of the order of diffusive times or more.

In several cases the convergence time limited by diffusion and impurity convection can be decreased by using multiplicators. These multiplicators increase the time derivatives of physical values by a factor defined by the user. The multiplicators for density of each sort of particles and for temperature equations can be chosen separately for each of four regions: two divertors, SOL above the X-point, core. No universal recommendation can be given about them. The choice of multiplicators is based on understanding of physically longest times and on the relative importance of different sorts of particles in nonlinear solution. For example, in the case of small impurity fraction ("trace impurity") the convergence of equations for this impurity can be accelerated without numerical instability. The multiplicators 10-100 can be recommended for equations for density of trace impurities in all regions. This approach is especially effective if the 
impurity distribution is calculated on the background of solution for the main ions already converged to steady state.

In the case of a deep transport barrier, the multiplicators 10-100 can be recommended for impurities, even if their density is considerable, inside the separatrix. The multiplicators 10 inside the separatrix for the main ions density and temperatures can be applied simultaneously with corresponding 10 times decrease of coefficients $\alpha_{\text {amain }}$ and $\alpha_{T}$ as described in section 3.2. The multiplicators 3-10 can be applied for impurity density in the SOL above the X-point if the slowest diffusion and parallel redistribution processes are there, but with care, since for significant impurity radiation such coefficients can lead to artificial oscillatory solution or no convergence of calculation. The non-linear solution in the divertor region is especially sensitive to the impurity accumulation and radiation, therefore no multiplicators for impurity and main ions density are recommended there in the case of considerable impurity fraction, the decrease of convergence time in this region is provided by modification of EIRENE sources by coefficient $\beta$

The impact of the effective sources and multiplicators on the time evolution of the calculation for ITER with neon and helium impurities and drifts turned on, is shown in Fig. 8. At time $2.207 \mathrm{~s}$ the neon puffing in initially steady state run was increased from $5.5 \cdot 10^{19}$ particles / $s$ to $8.0 \cdot 10^{19}$ particles $/ s$. Three computational schemes were applied: first, only partial flux surface averaging inside the separatrix was turned on in order to keep the time step $10^{-6} \mathrm{~s}$; second, the effective sources were applied additionally with the factor $\beta=50$; third, the effective sources were combined with the multiplicators, as given by table 1 . In the first scheme the density of the neon at the separatrix starts increasing but very slowly. The slope of the density tracing indicates characteristic time of more than 1 second, while misbalance of the puffing and pumping shows no evolution visible on the background of EIRENE noise. Estimating the time of puffing to pumping balance according to the section 3.3, the time necessary for ITER reaching steady state is about several seconds, which means more than $10^{6}$ iterations. Estimating a performance of 3 000-10 000 iterations in 24 CPU hours typical for modern computer clusters, this calculation will demand more than one year of CPU. In the case of effective sources turned on the characteristic time of puffing-pumping balance is 50 times smaller, about $20 \mathrm{~ms}$, which is seen in Fig.8a. The neon density at the separatrix at the initial stage increases in this case 20 times faster, Fig. $8 \mathrm{~b}$, as a result of bigger density in the divertor regions. Now the limiting factor for the convergence to the steady state is a diffusive time in the transport barrier, of about $100 \mathrm{~ms}$, which means the decrease of the CPU time by factor 10 comparing to computation without effective source. Still, CPU can be more than a month. The 
best scheme is application of both the effective sources and multiplicators. In this case the characteristic time can be further decreased, in the example case by factor 5, and solution can be obtained in $50 \mathrm{~ms}$, corresponding to less than a month of CPU, which is acceptable for the ITER research program.

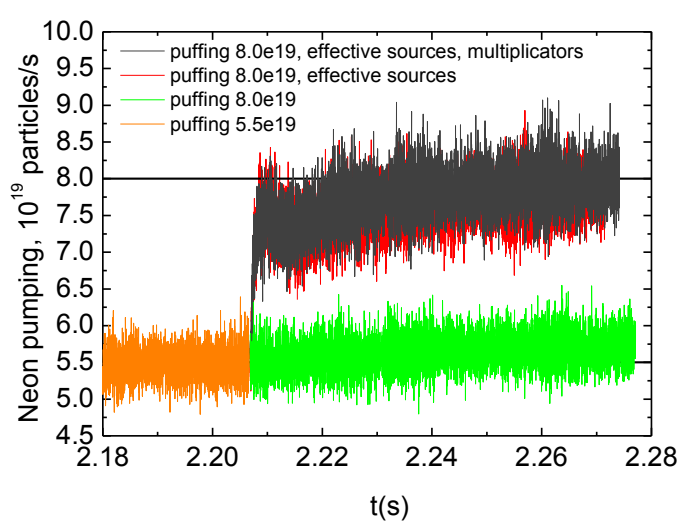

(a)

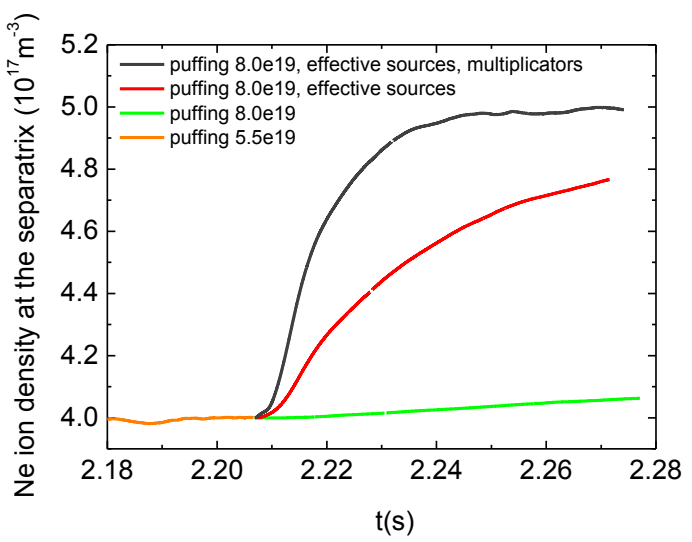

(b)

Fig.8. Time dependencies of parameters in calculations where the neon puffing was increased from $5.5 \cdot 10^{19}$ particles $/ s$ to $8.0 \cdot 10^{19}$ particles $/ s$.

(a) Neon pumping; (b) neon density at the separatrix.

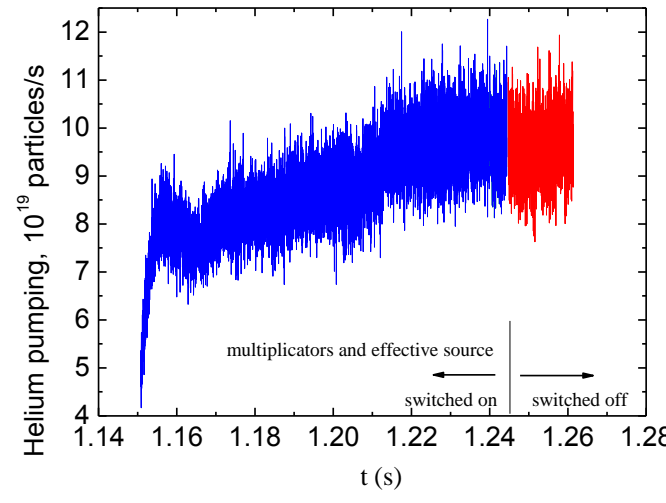

(a)

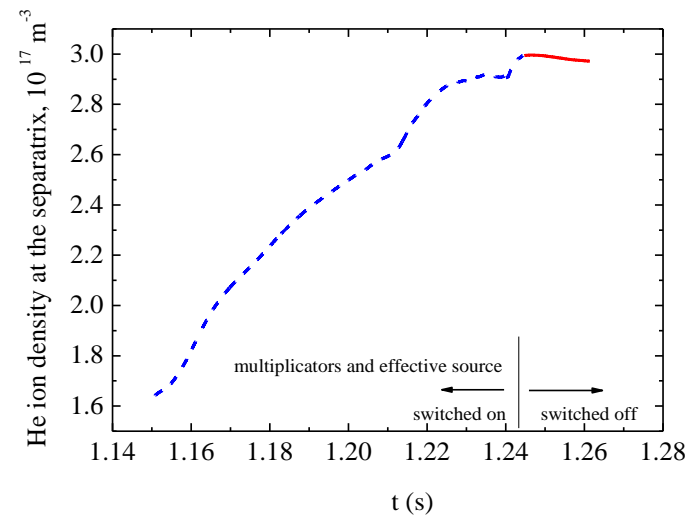

(b)

Fig.9. Time dependencies of parameters during the application of multiplicators and EIRENE source modification (1.15-1.245s) and after their switch off (after 1.245s). (a) Helium pumping; (b) helium density at the separatrix.

The characteristic times and comparative gains of the multiplicators and effective sources usage can be different in different cases, due to complexity of the system. In Fig.9 the coefficient $\beta=50$ and the multiplicators given in table 1 are applied at the beginning of calculations in which the flux of helium from the core was increased at $\mathrm{t}=1.15 \mathrm{~s}$ from $5 \cdot 10^{19}$ particles $/ \mathrm{s}$ to 
$10^{20}$ particles $/ \mathrm{s}$. Here the characteristic times are about twice bigger. The convergence is obtained in $100 \mathrm{~ms}$ with the time step $10^{-6} \mathrm{~s}$, demanding $10^{5}$ iterations. The calculations were performed here without drifts in order to avoid using the speed-up scheme described in section 2 and test the multiplicators and EIRENE source modification separately. After the approximate convergence at $\mathrm{t}=1.245 \mathrm{~s}$ the multiplicator coefficients and EIRENE source modifications were turned off. One can see that the balance of the puffing to pumping did not change during this final stage while the helium density at the separatrix shows the linear decrease with the characteristic time scale of about $2 s$, corresponding to an acceptable level of convergence.

\begin{tabular}{|l|l|l|l|l|}
\hline region & Deuterium & Neon & Helium & $\begin{array}{l}\text { Temperature } \\
\text { equations }\end{array}$ \\
\hline core & 10 & 100 & 100 & 10 \\
\hline SOL & 1 & 3 & 10 & 1 \\
\hline Inner divertor & 1 & 1 & 3 & 1 \\
\hline Outer divertor & 1 & 1 & 3 & 1 \\
\hline
\end{tabular}

Table 1. Multiplicators for calculations in Fig. 9.

\section{Conclusions}

Simulations of edge tokamak plasmas with the full SOLPS-ITER model including EIRENE neutrals and charged particle drifts are possible with acceptable convergence time scales only with implementation of improved numerical schemes. The most challenging limitation of convergence time step is associated with the poloidal redistribution of particles inside the separatrix by drifts of the geodesic acoustic mode nature. It can be overcome by implementation of one of two methods. The first one uses artificial slowing down of poloidal density and temperatures redistribution on the closed flux surfaces (partial flux surface averaging). In the second one (method of intermediate solution) the equations are modified so that they can be solved faster, with the fast solution very close to one we are seeking, as an initial approximation for convergence to the true solution.

The biggest characteristic times of the problem are times for balancing of puffing and pumping rates and different transport times. The time of puffing to pumping balancing can be decreased by introduction of an artificial particle source (method of artificial sources) into the problem. The transport times can be in several cases decreased by artificial multiplication of time derivatives for different equations in chosen separate regions. 
Implementation of these methods can speed up the modeling time by more than an order of magnitude. The best simulation strategy (including major choices that lead to very different codes) can be an important field for future work.

\section{Acknowledgement}

This work was carried out at Peter the Great St. Petersburg Polytechnic University under the support of the Russian Science Foundation, grant no. 17-12-01020. Numerical calculations were performed at the Polytechnic supercomputer center at Peter the Great St. Petersburg Polytechnic University. The views and opinions expressed herein do not necessarily reflect those of the ITER Organization.

\section{Reference}

[1] Kukushkin A S, Pacher H D, Kotov V, Pacher G W, Reiter D 2011 Fusion Engineering and Design 86 2865-2873

[2] Kotov V, Reiter D, Pitts R A, Jachmich S, Huber A, Coster D P and JET-EFDA contributors 2008 Plasma Phys. Control. Fusion 50105012

[3] Rozhansky V, Voskoboynikov S, Kaveeva E, Coster D, Schneider R, 2001 Nuclear Fusion 41387

[4] Reimold F, Wischmeier M, Potzel S, Guimarais L, Reiter D, Bernert M, Dunne M, Lunt T the ASDEX Upgrade team and the EURO fusion MST1 team 2017 Nuclear Materials and Energy 12193

[5] Rozhansky V, Kaveeva E, Molchanov P, Veselova I, Voskoboynikov S, Coster D, Counsell G, Kirk A, Lisgo S, ASDEX-Upgrade Team and MAST Team 2009 Nucl. Fusion 49025007

[6] Bonnin X, Dekeyser W, Pitts R, Coster D, Voskoboynikov S and Wiesen S 2016 Plasma Fusion Res. 111403102

[7] Wiesen S et al. 2015 J. Nucl. Mater.463 480

[8] Rozhansky V, Kaveeva E, Voskoboynikov S, Coster D, Bonnin X, Schneider R 2002 Phys. Plasmas 9 3385-3394 
[9] Rozhansky V, Kaveeva E, Veselova I, Voskoboynikov S, Coster D 2016 Contributions to plasma physics $\mathbf{5 6} 587-591$

[10] Rognlien T D, Milovich J L, Rensink M E, Porter G D 1992 J. Nucl. Mater. 196-198 347

[11] Winsor N, Johnson J L and Dawson J M 1968 Phys. Fluids 112448

[12] Hirshman S P, Sigmar D J 1981 Nucl. Fusion 211079

[13] Helander P, Sigmar D J, Collisional transport in magnetized plasmas, Cambridge University Press 2002 Cambridge

[14] Rozhansky V, Kaveeva E, Voskoboynikov S, Coster D, Bonnin X, Schneider R 2002 Nucl. Fusion 42 1110-1115

[15] Sytova E, Senichenkov I, Kaveeva E, Rozhansky V, Veselova I, Voskoboynikov S, Coster D 2016 Proc. $43^{\text {th }}$ EPS Conf. on Controlled Fusion and Plasma Physics Lisbon ECA Vol. 40E P1.054 


\section{Appendix}

Here the practical recommendations are summed up for use of speed up schemes.

\section{Method of intermediate solution}

Restrictions and application limits:

- The new numerical scheme has been successfully tested on ITER cases with one main (hydrogenic) species plus impurities with kinetic neutrals.

- The scheme provides robust initial approximation for normal code convergence towards the final solution. This initial approximation can be discussed as one very close to solution in SOL and divertor region, and can be used for analysis of physical processes in these regions.

- The scheme demands final stage of calculation without modification of coefficients consuming about half of the CPU time if accurate description of the flows inside the separatrix is of interest.

Practical recommendations

\section{Step one}

1) In b2mn.dat increase the time step from $2 \cdot 10^{-8} \mathrm{~s}$ to $10^{-6}$ :

\section{'\#b2mndr_dtim' '2.0e-8' \\ 'b2mndr_dtim' '1.0e-6'}

2) In b2mn.dat increase the value of anomalous conductivity $\sigma_{A N}$ from $10^{-7} e n_{e}$ to $4 \cdot 10^{-6} \mathrm{en}_{e}$ :

Instead of

*cfsig (0)

(1)

(2)

(3)

(4)

(5)

(6)

'-1' $1.0 \mathrm{e}-07 \quad 0.0 \mathrm{e}+00 \quad 0.0 \mathrm{e}+00 \quad 0.0 \mathrm{e}+00 \quad 0.0 \mathrm{e}+00 \quad 0.0 \mathrm{e}+00 \quad 0.0 \mathrm{e}+00 \quad 0.0 \mathrm{e}+00$

*cfalf $\quad(0)$

(1)

(2)

(3)

(4)

(5)

(6)

'-1' $1.0 \mathrm{e}-07 \quad 0.0 \mathrm{e}+00 \quad 0.0 \mathrm{e}+00$

$0.0 \mathrm{e}+00$

$0.0 \mathrm{e}+00 \quad 0.0 \mathrm{e}+00 \quad 0.0 \mathrm{e}+00 \quad 0.0 \mathrm{e}+00$ set the following
*cfsig (0)
(1)
(2)
(3)
(4)
(5)
(6)

$\begin{array}{llllllllllll} & -1 & 4.0 \mathrm{e}-06 & 0.0 \mathrm{e}+00 & 0.0 \mathrm{e}+00 & 0.0 \mathrm{e}+00 & 0.0 \mathrm{e}+00 & 0.0 \mathrm{e}+00 & 0.0 \mathrm{e}+00 & 0.0 \mathrm{e}+00\end{array}$
*cfalf (0)
(1)
(2)
(3)
(4)
(5)
(6)
$\begin{array}{llllllllll} & -1 & 4.0 \mathrm{e}-06 & 0.0 \mathrm{e}+00 & 0.0 \mathrm{e}+00 & 0.0 \mathrm{e}+00 & 0.0 \mathrm{e}+00 & 0.0 \mathrm{e}+00 & 0.0 \mathrm{e}+00 & 0.0 \mathrm{e}+00\end{array}$

3) In b2mn.dat increase the viscosity coefficient by a factor of 20 : 


\section{'\#b2trcl_cvsa_mltpl' '1.0' by default \\ 'b2trcl_cvsa_mltpl' '20.0'}

4) Calculate to converged state

\section{Step two}

Return the initial values of coefficients and continue the calculations from the state obtained on step 1, for about 50000 iterations

\section{Method of partial flux surface averaging}

Restrictions and application limits:

- The application of new numerical scheme converges to the same steady state solution as one can get without this scheme, i.e. after reaching the converged steady state solution no additional run with modified input parameters is needed.

- The new numerical scheme has been successfully tested on cases with one main (hydrogenic) species and fluid neutrals (no impurities).

- The new numerical scheme has been successfully tested on cases with one main (hydrogenic) species plus impurities with kinetic neutrals.

- The scheme should not be applied if the strong poloidal density and/or temperature variation are expected in the solution, e.g. for detached plasma with cold X-point.

Practical recommendations

In b2mn.dat increase the time step:

- for ASDEX-Upgrade

$\begin{array}{lc}\text { '\#b2mndr_dtim' } & \text { '1.0e-7' } \\ \text { 'b2mndr_dtim' } & \text { '1.0e-5' } \\ \text { • for ITER } & \\ \text { '\#b2mndr_dtim' } & \text { '1.0e-8' } \\ \text { 'b2mndr_dtim' } & \text { '1.0e-6' }\end{array}$

In b2mn.dat set up the desired minimal density value

\section{'b2mndr_na_min' '1.0e8'}

In b2.numerics.parameters set up the control parameters $\alpha_{a}, \alpha_{T}$ for each species and for temperature (example is given for $\mathrm{D}+\mathrm{N}$ plasma)

$$
\begin{aligned}
& \text { corr_core_dn }(0)=10 * 0.01 \text {, } \\
& \text { corr_core_dt }=0.01,
\end{aligned}
$$

Here corr_core_dn stands for $\alpha_{a}$ and corr_core_dt stands for $\alpha_{T}$. 


\section{Method of effective source}

Restrictions and application limits:

- The new numerical scheme has been successfully tested on ITER cases with one main (hydrogenic) species plus impurities with kinetic neutrals.

- The application of new numerical scheme converges to the same steady state solution as one can get without this scheme, i.e. after reaching the converged steady state solution no additional run with modified input parameters is needed.

Practical recommendations

In b2mn.dat set the numerical coefficient $\beta$ :

\section{'\#b2stbr_sna_corr' '0.0' by default \\ 'b2stbr_sna_corr' '50.0'}

